\title{
Progress in Operational Analysis of Launch Vehicles in Nonstationary Flight
}

\author{
George James, Mo Kaouk, Tim Cao \\ Loads and Structural Dynamics Branch \\ NASA Johnson Space Center \\ Houston, Texas 77058
}

\begin{abstract}
This paper presents recent results in an ongoing effort to understand and develop techniques to process launch vehicle data, which is extremely challenging for modal parameter identification. The primary source of difficulty is due to the nonstationary nature of the situation. The system is changing, the environment is not steady, and there is an active control system operating. Hence, the primary tool for producing clean operational results (significant data lengths and data averaging) is not available to the user. This work reported herein uses a correlation-based two step operational modal analysis approach to process the relevant data sets for understanding and development of processes. A significant drawback for such processing of short time histories is a series of beating phenomena due to the inability to average out random modal excitations. A recursive correlation process coupled to a new convergence metric (designed to mitigate the beating phenomena) is the object of this study. It has been found in limited studies that this process creates clean modal frequency estimates but numerically alters the damping.
\end{abstract}

\section{KEYWORDS}

Operational Modal Analysis, Signal Processing, Hilbert Transform, Correlation Function, Damping Estimation

\section{INTRODUCTION}

The spacecraft launch environment is a highly complex and non-stationary event that is characterized by high amplitude input forces, highly variable loads, a wide spectrum of responses, constantly changing vehicle mass, active control interactions, staging, and limited instrumentation. At the same time, structural response analyses and loads estimations must be performed with models that are only partially validated using ground test data due to the fact that access to diagnostic and environmental ground tests are limited. To compound matters, project managers tend to reduce uncertainty factors designed to protect for loads increases and model unknowns. As a result, the designs progress rapidly before loads and structural problems are uncovered. This means that there are very few tools available to recover from structural dynamics issues in such a highly dynamic environment without costly redesigns late in the design cycle or in early operations.

Traditional modal testing applies a known input to the structure (or components/test articles) and structural dynamic parameters are then extracted. In spite of the relatively pristine data, these dedicated tests are often difficult to schedule or afford (and rarely achieve flight-like loading conditions). The inclusion of operational modal testing and analysis tools can be used to offset these limitations by providing additional cost and schedule effective opportunities for diagnostic information extraction. These opportunities are available on the ground and during flight as well as on full-up systems, subsystems, components, and test articles.

\section{TECHNICAL BACKGROUND}

The technology for extracting structural dynamic properties from structures (i.e. modal testing) has rapidly improved over the last several decades. Operational modal analysis (OMA) is one of the specialized spin-offs that have grown in importance over the last 20 years. In OMA, a known and measured input is not artificially imparted to the structure to drive the known responses but measurements are made in-situ and processed to obtain a subset of the desired modal data. This approach is very useful for large in-service structures that cannot be removed from service effectively (e.g. bridges, buildings, wind turbines, off-shore structures, etc.). One of the earliest OMA techniques was the Natural Excitation Technique (NExT). ${ }^{1}$ This development showed that for a class of inputs, the auto and cross-correlation functions could be processed as time decay functions to estimate the modal frequencies and modal damping properties. Time domain estimators, such as Polyreference ${ }^{2}$ or the Eigensystem Realization Algorithm (ERA) ${ }^{3}$ have been used to process such data. Appendix A contains the theoretical 
background for this technique. Recent years have seen the OMA field become rich with other advanced techniques having broad applicability. ${ }^{4}$ However, NExT has continued to spawn on-going efforts to improve, expand, and further understand the approach. ${ }^{5,6,7,8,9,10}$

\section{Alternative Approaches for Traditional OMA}

There are two general classes of algorithms for performing stationary linear OMA: (1) time history-based techniques that are generally related to Stochastic Subspace Identification (SSI) and frequency domain-based techniques that are related to Frequency Domain Decomposition (FDD). ${ }^{1,411,12,13,14}$ Early time domain approaches included tools like the Random Decrement and Maximum Entropy Methods. ${ }^{15,16,17,18}$ The technical basis for the NExT approach mentioned previously involved converting measured responses into auto and cross-correlation functions and processing with standard time domain modal analysis routines. ${ }^{1}$ However, the more general SSI techniques directly integrate the correlation calculations and modal processing algorithms into a single step rooted in discrete time system identification theory. ${ }^{12,14}$ The earliest manifestations of FDD were peak picking and half-power bandwidth estimation schemes operating on the Power Spectral Density (PSD) functions. ${ }^{13}$ However the advanced FDD algorithms refine the modal parameter estimates using powerful tools like the Singular Value Decomposition (SVD). ${ }^{4,13}$ An interesting direction for frequency domain approaches involves the use of Hilbert transforms applied to PSD's to obtain biased Frequency Response Function (FRF) estimates. ${ }^{4,19}$

There is another direction in operational testing that involves estimating the forces acting on the system. This would allow more traditional FRF-based approaches to be used for system identification. These forces can be estimated via known mass changes to the system or via hybrid analytical/experimental data. ${ }^{20,21}$ For non-stationary systems, Wavelet Analyses represents one possible approach. ${ }^{22,23}$ Another possible approach is via the Wagner-Ville developments. ${ }^{24}$ For non-linear and non-stationary systems, the empirical Hilbert-Huang method is a possibility. ${ }^{25,26,27}$

\section{Launch Environment Analyses}

There have been a limited number of reported attempts to analyze flight data to extract modal parameter information, although there are certainly many other unreported attempts. The time domain approaches based on correlation and SSI are generally used for flight data analyses as the rapidly changing vehicle properties do not allow the full advantages of the frequency-domain approaches to be realized. The responses are generally broken into a series of time windows, each of short duration (and quite often significant overlap), that are processed individually. If the loading and system characteristics are fairly constant over each window, then estimates of the changing parameters can be obtained as a function of flight time. $28,29,30,31,32,33,34,35$

Three of the references listed above show that one trajectory for NExT has been to act as a pathfinder for the development of operational analysis techniques to process launch data. ${ }^{28,29,30}$ This effort is doubly challenging as modal damping is one of the most sought after parameters from the launch environment, which is difficult to extract even in well controlled stationary environments. However, flight damping during launch becomes a critical part of the discussions during the design and operations of space vehicles due to the control over the response of structures and components. Hence, modal damping has become an important metric for the utility flight data and flight data analyses.

Reference [28] discusses the earliest work in the application of the NExT/OMA approach to launch data from a missile-based system. The work used tight narrow-band filters to limit the data under processing to one or two modes at time. Also, the time windows generally covered two to four cycles of the modes and had significant overlap. In order to make this analysis tenable, the modal engine $\left(\mathrm{ERA}^{3}\right)$ had to be called automatically. Even with tight filtering and the processing software able to call ERA when needed, this analysis was a very labor intensive process. The manual effort involved assessing the results 
of the processing for each window, making decisions on the selected roots, resetting parameters if required, and restarting the processing when needed. However, the traces for modal frequency and damping look fairly reasonable and smooth (except for the first mode damping in first stage flight).

Reference [29] discusses a later launch analysis from the Space Shuttle. For this analysis and automation process called AUTO-ID was added to the tool. ${ }^{36,37}$ The addition of this technique eased the computational burden of extracting parameters in a consistent manner from the multitude of correlation functions calculated from the sliding time window segments of the random time histories to allow a rapid assessment of the data. This was a much less labor-intensive process that the original processing effort as discussed in Reference [28]. As a result many more modes were assessed in much less time than seen in the previous case study. However, the results were not a pristine and more excursions in the frequency and damping were allowed as a result. The reported data for modal damping still shows trends and excursions during flight.

Reference [30] is a recent study performed on the PA-1 test flight. This test flight provided a very challenging data set with a very short flight time and extremely rapidly changing modal frequencies. Autonomous identification was not used and the amount of frequencies studied was less that the study discussed in Reference [29]. The user effort required was intermediate between the two previous studies discussed. Since the user interacted with data to a greater extent than previously and the experience of the previous exercises was available, two problems were for immediate attention: beat-like phenomena in the correlation functions of short time records and potential interactions between the vehicle control systems and extracted damping in the lowest bending modes of the vehicles. A working hypothesis for the first problem (beat-like phenomena) has been developed in which the phenomena are apparent increases in correlation due to the lack of ability to temporally average out the random characteristics of the responses. A limited number of potential mitigations for this effect are in-hand as well plans to assess other non-correlation based approaches. This work continues an effort to study a mitigation which uses multiple correlation processes as applied to short time histories. ${ }^{38}$ The second problem of potential control system interactions is still being scoped.

\section{STUDY OF RECURSIVE CORRELATION AND AN ASSOCIATED CONVERGENCE METRIC}

A most significant complexity associated with operational analysis of launch systems is the unsteadiness due to rapidly changing mass properties. This usually drives the available time records to be very short due to the need to utilize some type of sliding window analysis (at least for a process that assumes stationarity). The analysis of several recent data sets has shown that one effect (at least on the correlation-based processing approaches) is a "beating" or "blooming" phenomena which limits the amount of the correlation functions that can be used for processing. ${ }^{30,35,39}$ Although the first low-lag points in the correlation functions are relatively unaffected, the higher-lag time data points are relatively useless for analysis.

\section{Previous Findings}

Figure 1 illustrates the beating phenomena as illustrated by a simple one DOF analytical model of a $10 \mathrm{~Hz}$ mode with $1 \%$ damping excited by random white noise using Newmark-Beta integration. The 32,768 length time history has a time step of .001 seconds. The random input is shown in the top plot of Figure 1. The displacement response of the $10 \mathrm{~Hz}$ system is shown in the middle plot. Displacement is used as opposed to the more easily measured acceleration as it illustrated the issue with more clarity. Notice that the response shows random excitations of the $10 \mathrm{~Hz}$ system mode, which eventually damps out. The lower plot provides the autocorrelation function of the displacement shown in the middle plot. The beating phenomena are clearly seen as the correlation increases at longer lags. Note that the "beating" terminology is adopted as the correlation function looks like a time history of closely spaced modes interacting or "beating". The periodic increases in correlation deviates from the theoretical damped sinusoids that are expected from OMA/NExT correlation functions and limit the utility to separate closely space modes as only early lags can be used for analysis. ${ }^{1}$ The working hypothesis for this phenome- 
na is that the random "blooms" in the response data (middle plot of Figure 1) result as the internal modes are randomly excited by the input. During the correlation process these "blooms" in the response data become the beating phenomena in the correlation functions (see bottom plot of Figure 1).

Previous work to address this issue suggested that the beating effects can be reduced via repeated correlation calculations using the same parent time data. Figure 2 shows the effectiveness of this approach. The top plot shows an autocorrelation function of a $10 \mathrm{~Hz}$ single DOF system excited randomly. If a another correlation calculation is performed using the first autocorrelation function as the parent data then the correlation function shown in the middle plot results. The bottom plot results after performing an additional eight correlation calculations using the function shown in the middle plot as the parent data (10 correlation calculations total). This obviously produces a damped sinusoidal function as expected. It can be seen that the proper damping seemed to result after a number of these correlations are performed. However, the application of additional correlations does numerically alter the extracted damping. Hence, this approach needs a convergence metric to allow the analyst to know when to stop performing additional correlations. ${ }^{30}$ A metric was presented in reference [38] and will be repeated here. However the ongoing effort has discovered that there are numerical issues with the recursive correlation approach that need to be understood as validation of the technique is proving elusive.

\section{Development of a Convergence Metric}

The problem at hand involves performing additional specialized averaging (in the form of autocorrelation functions) on the randomly excited data. The desired result is a mathematical function that more closely matches the theoretical expectation of damped sinusoids. The primary issue in the current single pass correlation functions is not the sinusoidal content but the incomplete capture of the decaying exponential envelope. Hence, the first step will be to extract the envelope. To do this we start by generating an analytic function using the Hilbert transform of the first pass correlation functions:
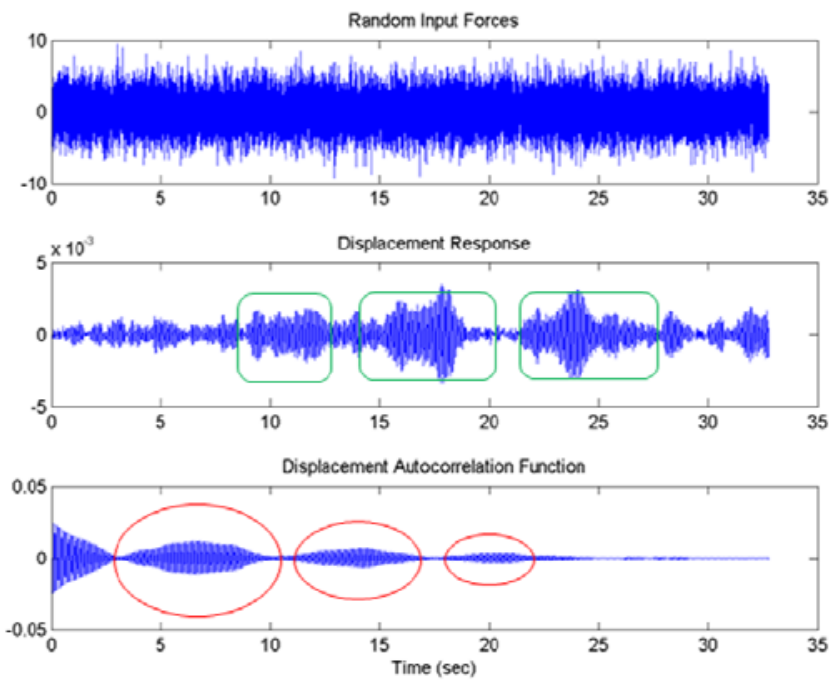

Figure 1. Beating Phenomena in Correlation Functions in Single DOF Analytical Data 

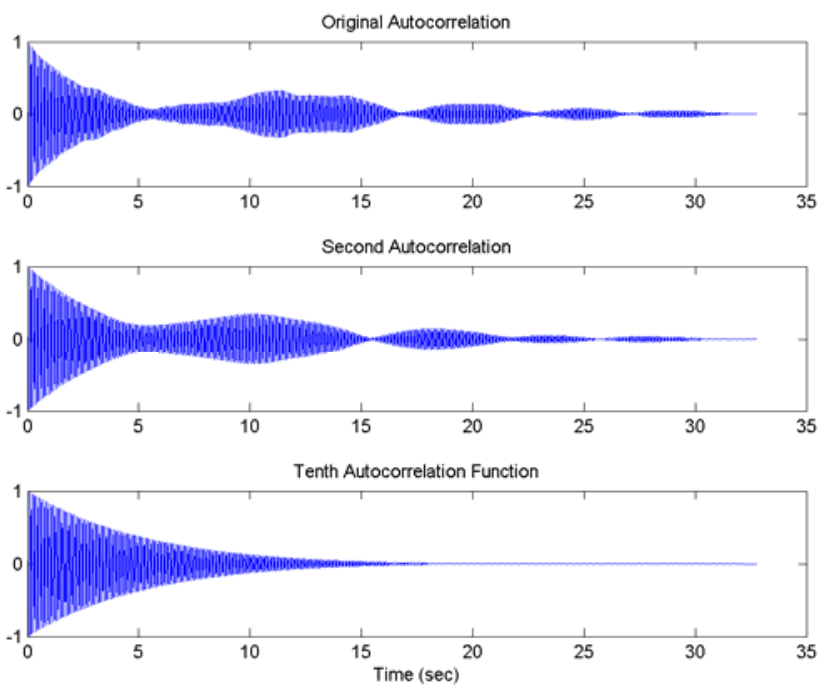

Figure 2. Use of Repeated Correlation Calculations to Reduce Beating Phenomena

$$
C_{i j}(t)=R_{i j}(t)+\sqrt{-1} * H_{i j}(t) ; \text { where }
$$

$R_{i j}$ is correlation function between outputs $i$ and $j$;

$H_{i j}$ is the Hilbert transform of $R_{i j}$; and

$C_{i j}$ is the analytic function associated with $R_{i j}$ and $H_{i j}$.

The Hilbert transform is defined as follows:

$$
H_{i j}(t)=\frac{1}{\pi} \int_{-\infty}^{\infty} \frac{R_{i j}(\tau)}{(t-\tau)} d \tau .
$$

The envelope is then the amplitude of the complex analytic function:

$$
A_{i j}(t)=\sqrt{R_{i j}^{2}(t)+H_{i j}^{2}(t)} .
$$

Figure 3 contains an example of the previous functions. The top plot is the numerical displacement response of a singlemode $10 \mathrm{~Hz}$ system with $1 \%$ damping as excited with random inputs. The middle plot shows the autocorrelation function 
from the data in the top plot. The bottom plot shows the associated envelope as described by equation (3) above. Now the theoretical result suggests that the correlation function looks a decaying sinusoid, hence for this single-mode system, the envelope should look like an exponential decay:

$$
A_{i j}(t) \approx A_{0} \mathrm{e}^{-a t} .
$$

Therefore for this simplified system, we can take the natural log to simplify:

$$
\ln \left(A_{i j}(t)\right) \approx \ln \left(A_{0}\right)+\ln \left(e^{-a t}\right)=a_{0}-a t .
$$

There are two possible approaches to determine estimates of the exponential parameter from the log envelope. The first approach is to fit a line to the natural $\log$ of the envelope with the offset providing $a_{0}$ and the slope $(a)$ parameter. For subsequent discussions, this approach shall be referred to as the "linear slope" approach and the slope parameter designated as " $a$," The alternative approach is to take a mean value of the differences between any two values of the log envelope and divide by the time step. This approach will be referred to as the "mean difference" and the slope parameter designated as " $a_{2}$ ". Figure 4 shows those two approaches and the best fit estimates for the first half of the records of the log envelope given in Figure 3.
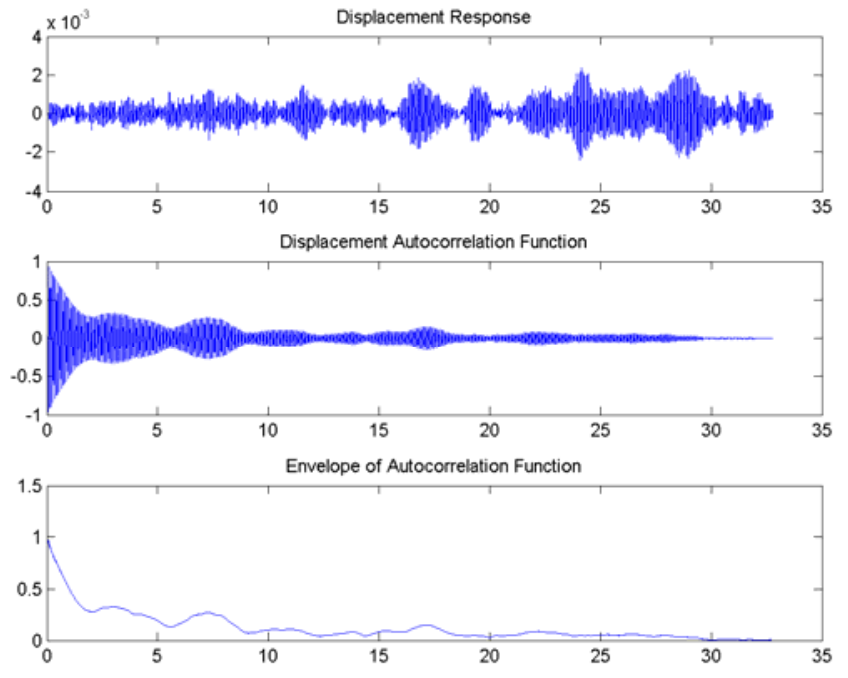

\section{Figure 3. Envelope of Correlation Function of a Single DOF Analytical Data}

In order to further increase the content of the modal response and reduce the effects of the random forcing function, the current correlation function is subjected to another pass through the correlation processing step. Correlation processing (in a time domain sense) involves a summation process of all data separated by the same number of time steps (or correlation lags in this case): 

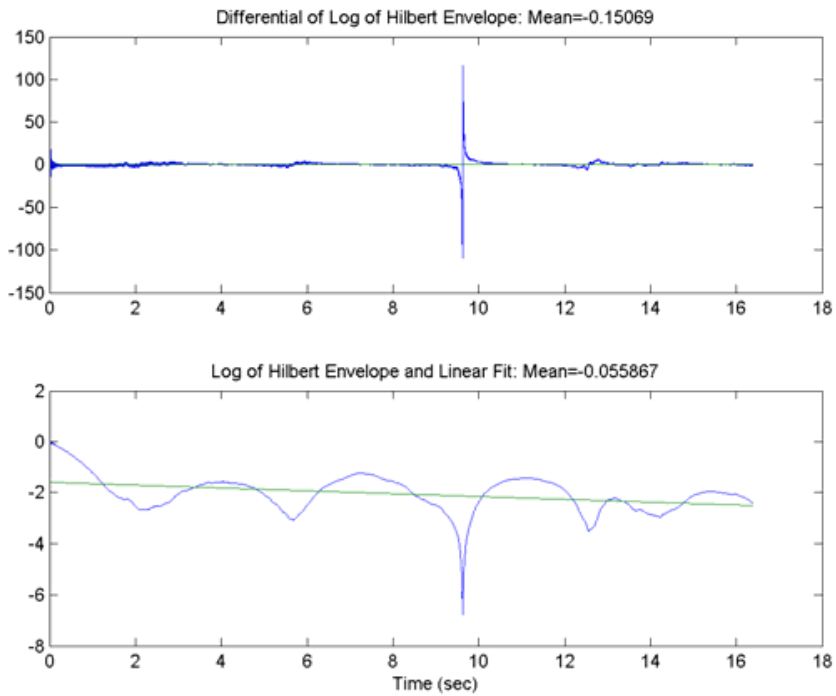

Figure 4. Fits to Log Envelope of a Single DOF Analytical Correlation Function

$$
\begin{array}{ll}
R_{i j}(\tau)=\sum_{t=0}^{t_{\max }-\tau-1} x(t+\tau) y(t) & \tau \geq 0 \\
R_{i j}(\tau)=R_{i j}(-\tau) & \tau<0
\end{array}
$$

Now if we substitute the original time history products for the current correlation function and add a subscript to denote the iteration or number of the successive correlation steps $(k)$ :

$$
\begin{array}{ll}
R_{i j k}(\tau)=\sum_{t=0}^{t_{\max }-\tau-1} R_{i j l}(t+\tau) R_{i j l}(t) & \tau \geq 0 \\
R_{i j k}(\tau)=R_{i j k}(-\tau) & \tau<0
\end{array}
$$

Where $l=k-1$.

Hence, the variables of interest will be estimated at each iteration and will receive a " $k$ " subscript: $H_{i j k}(t), C_{i j k}(t), A_{i j k}(t), a_{0 k}$, $a_{1 k}$, and $a_{2 k}$. The slope parameters are updated each iteration and compared to the previous value and scaled with respect to the first slope value to produce a convergence metric (labeled as $b_{0 k}$ and $b_{1 k}$ ). Hence for the linear slope metric: 


$$
b_{1 k}=100 *\left(\frac{a_{1 k}-a_{1 l}}{a_{11}}\right) \text {. }
$$

And for the mean difference metric:

$$
b_{2 k}=100 *\left(\frac{a_{2 k}-a_{2 l}}{a_{21}}\right) \text {. }
$$

Figure 5 shows typical convergence histories for these two metrics. The top plot provides the actual values of the slope parameters (as illustrated in Figure 4) for multiple subsequent correlation iterations. The lower plot shows the traces of the related convergence metrics. For this example the process was ended when the convergence metric was lower than .1\% in either parameter. Typically the mean difference converges first. Figure 6 shows the fits to the converged log envelopes. Although there are some obvious numerical issues for longer lag times, the overall trends are much closer to the expected exponential decay model. Figure 7 shows the final correlation trace after convergence in the top plot. The middle plot contains the final envelope of the correlation trace. These plots show the expected decaying exponential shape. After performing a modal identification on the data shown in Figure 7, the resulting modal frequency is found to be $10.1 \mathrm{~Hz}$ with $.99 \%$ damping. The resulting synthesis to the converged data is provided in Figure 8. The final extracted frequency and damping is used to generate damped sine and cosine functions which are least-squares fit to the correlation data to generate the synthesis.
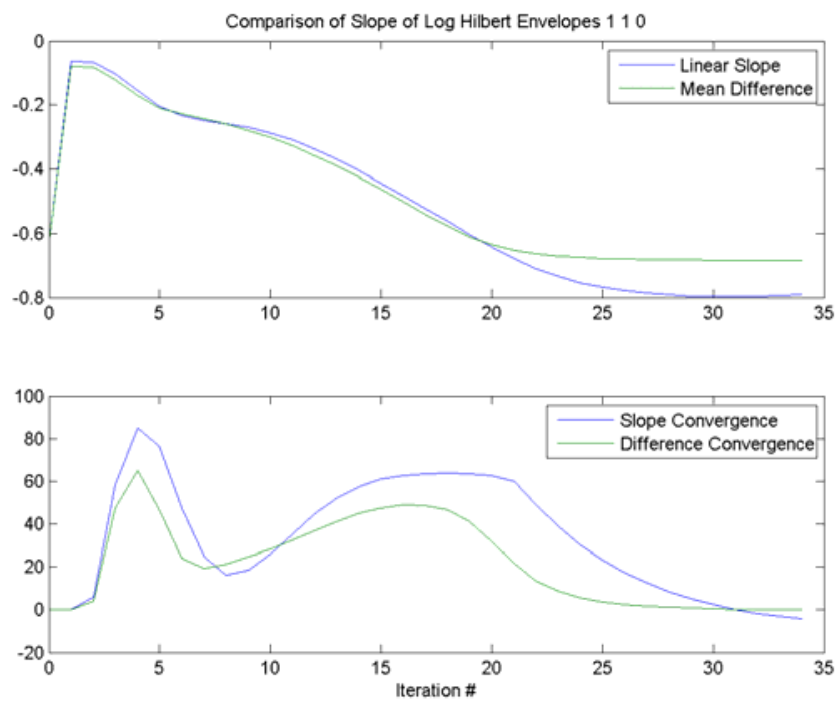

Figure 5. Convergence of Multiple Correlations of Single DOF Analytical Data 

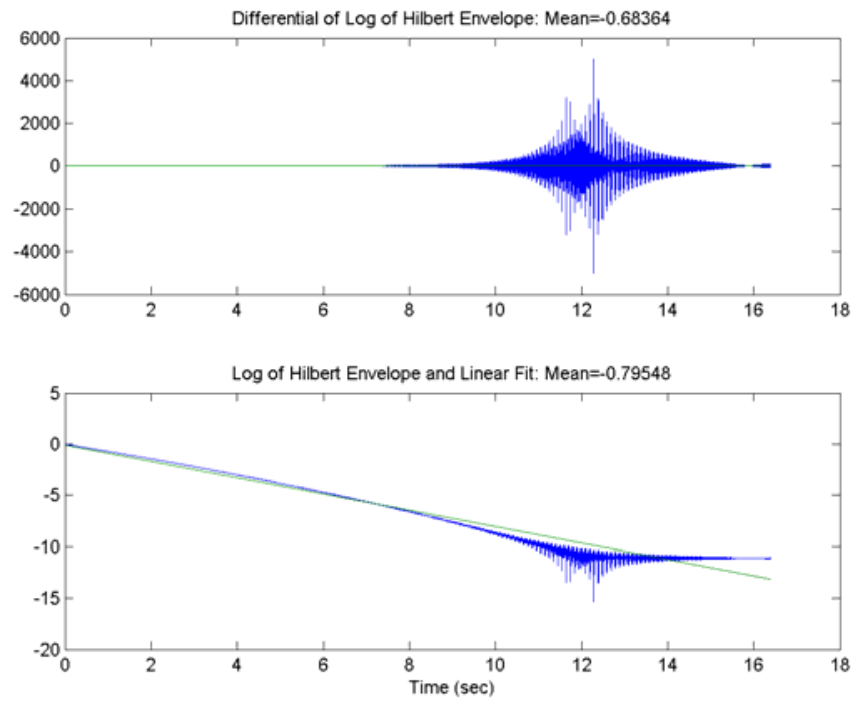

Figure 6. Converged Fits to Log Envelope of Single DOF Analytical Data
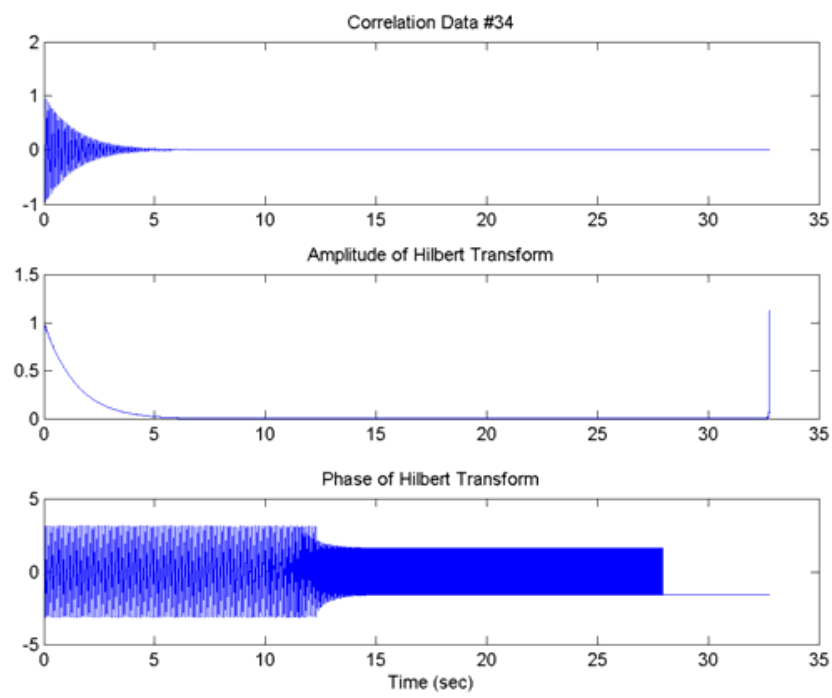

Figure 7. Envelope of Converged Correlation Function of Single DOF Analytical Data 

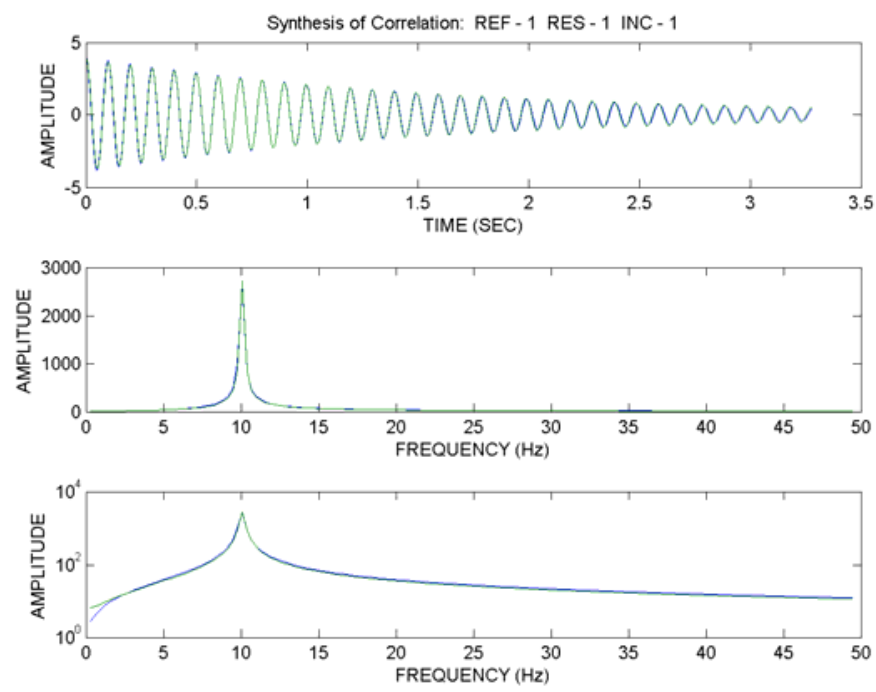

\section{Figure 8. Data and Synthesis of Modal Fit to Converged Correlation of Analytical Data}

Numerical Assessment of Recursive Correlation/Convergence Metric Approach

The data provided above to study and develop a mitigation strategy for correlation beating on short time records is subject to several simplifying factors that will have to be assessed before the process becomes truly useful. These include multiple modes, data stationarity, multiple sensors, numerical round-off error, system noise, the role that the phase of the analytic function may play, digital parameter shifting (as seen in numerical integration algorithms), and the order to apply the crosscorrelation and iterative autocorrelations when using data from different sensors. However, the most critical unknown to be removed before this mitigation process is useful for short time record launch data analysis are the actual effects of data record length. Hence, a scoping study of the effects was performed some critical features of the overall technique were uncovered.

This scoping study involved utilizing several different data record lengths of data to develop estimates of the modal frequency and modal damping to assess the trends in the parameters. For the $10 \mathrm{~Hz}$ analytical data with $1 \%$ damping, data lengths from 1024 samples to 32768 length data record were assessed in increasing data record length increments of 1024 samples for 32 different analyses. Figure 9 provides the results of this study. The top plot shows the variation of the extracted frequency as the data record length processed in increased. The frequency variations are exaggerated due to the scale and are not significantly problematic. However, the lower damping plot shows a clear trend of increasing damping estimates away from the $1 \%$ expected value as the record length is increased. This is an issue that must be dealt with as damping is a critical parameter in these studies and the shorter time records are the region that launch data processing will be utilizing. The good news from the initial study was that the process break-down appeared to be stochastic with a clear monotonic trend. This was the state of the work as reported in Reference [38].

Further work to understand short time history effects lead to an unfortunate finding. Figure 10 shows the same scoping study if the damping is increased from $1 \%$ to $10 \%$. In this study, the damping was seen to continue to converge to $1 \%$. Several 
other input values of damping were exercised with very similar results. Hence the extracted damping values of the recursive correlation technique were completely numerical in origin.

\section{Analytical Study of Recursive Correlation Damping}

In order to study the short time history and recursive correlation effects, assume a damped cosine function (see Appendix 1 for the theoretical basis of this assumption):

$$
x(t)=\theta^{-\delta \omega} \operatorname{cog}(\omega t)
$$

The autocorrelation function could then be defined as:

$$
F_{w}(\beta)=\int_{\sigma_{1}}^{\alpha_{\alpha}} x(\alpha+\beta) \alpha(\sigma) d \sigma
$$

With the following definitions feeding equation (11):

$$
\begin{aligned}
& \alpha=\delta \omega t \\
& \beta=\hat{\omega} \omega \\
& x_{1}=0 \\
& \alpha_{2}=\delta \omega\left(t_{f}-\tau\right)=\alpha_{f}-\beta \\
& x(\alpha)=e^{-\alpha} \cos \left(\frac{\alpha}{8}\right) ; \text { and }
\end{aligned}
$$

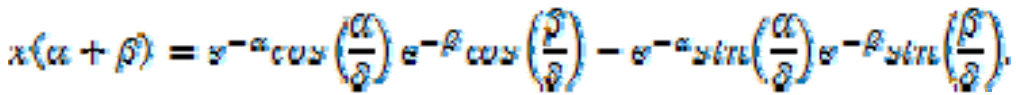

After rearranging the following equation results:

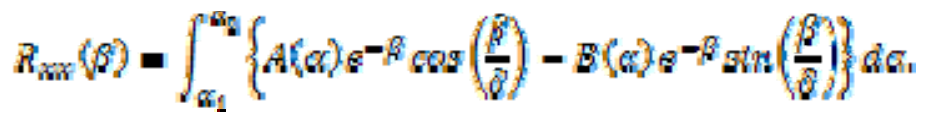

With the following definitions used to generate equation (18):

$$
\begin{gathered}
A(\alpha)=\frac{1}{2} \theta^{-2 \kappa}\left[1+\cos \left(\frac{2 \alpha}{8}\right)\right], \text {, }[n d \\
B(\alpha)=\frac{1}{2} \theta^{-2 \alpha} \sin \left(\frac{2 \alpha}{8}\right)
\end{gathered}
$$



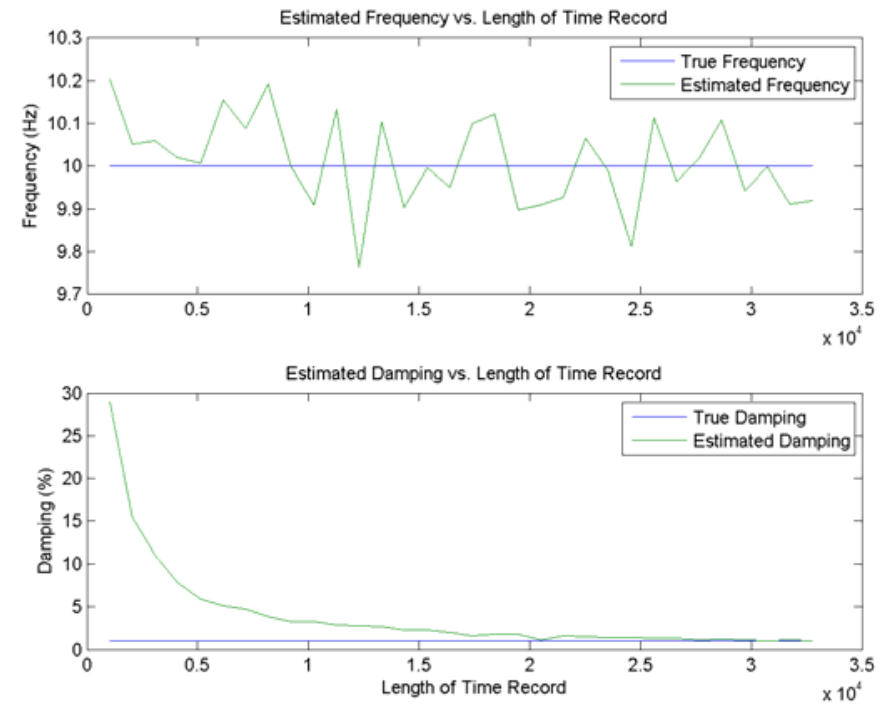

Figure 9. Effect of Data Record Length using Single DOF Analytical Data - 1\% Damping
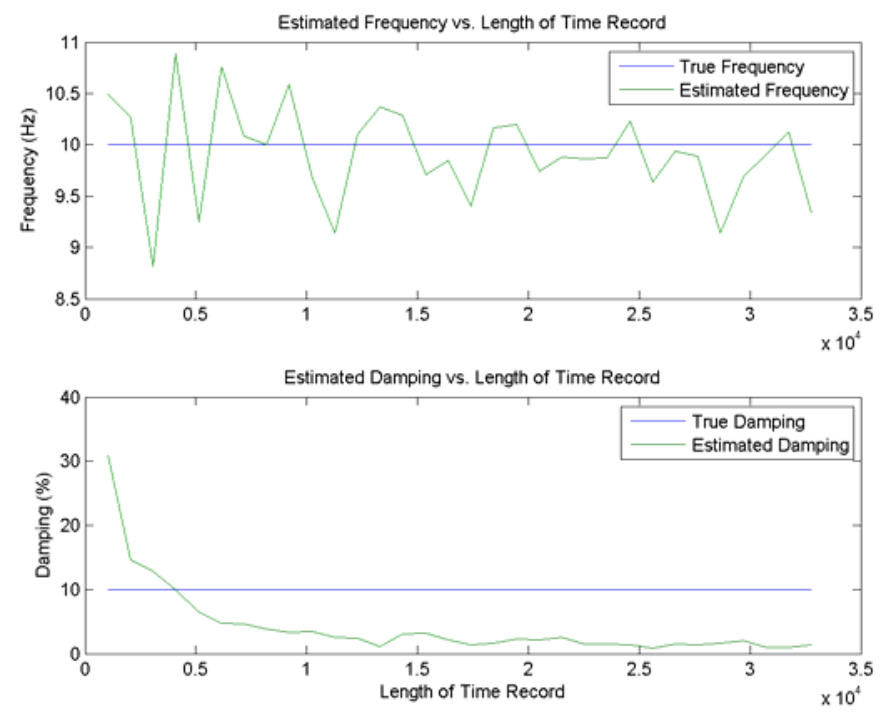

Figure 10. Effect of Data Record Length using Single DOF Analytical Data - 10\% Damping 
Now when the following assumption is used:

$$
0^{2} \approx 0,
$$

These simplifications result:

$$
\begin{aligned}
& \int_{-\alpha_{4}}^{\alpha_{n}} A(\alpha) d \sigma \approx \frac{1}{4}\left(1-2^{-2 \sigma_{n}}\right)+0 \text {; and } \\
& \int_{\sigma_{6}}^{\pi_{n}} B(\alpha) d \alpha \curvearrowright \alpha_{1}
\end{aligned}
$$

With these simplifications, the autocorrelation reduces to the following:

$$
R_{X X}(\beta) \propto \frac{1}{4}\left[1-\theta^{-2(\alpha f-\beta)}\right] \theta^{-\beta} \cos \left(\frac{\beta}{\delta}\right)
$$

Equation (24) can also be written as the following:

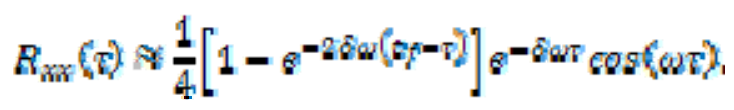

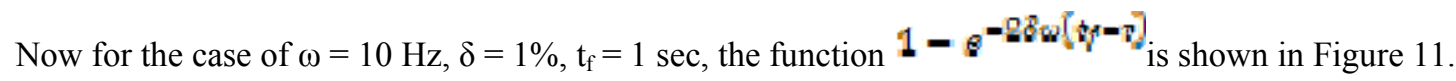

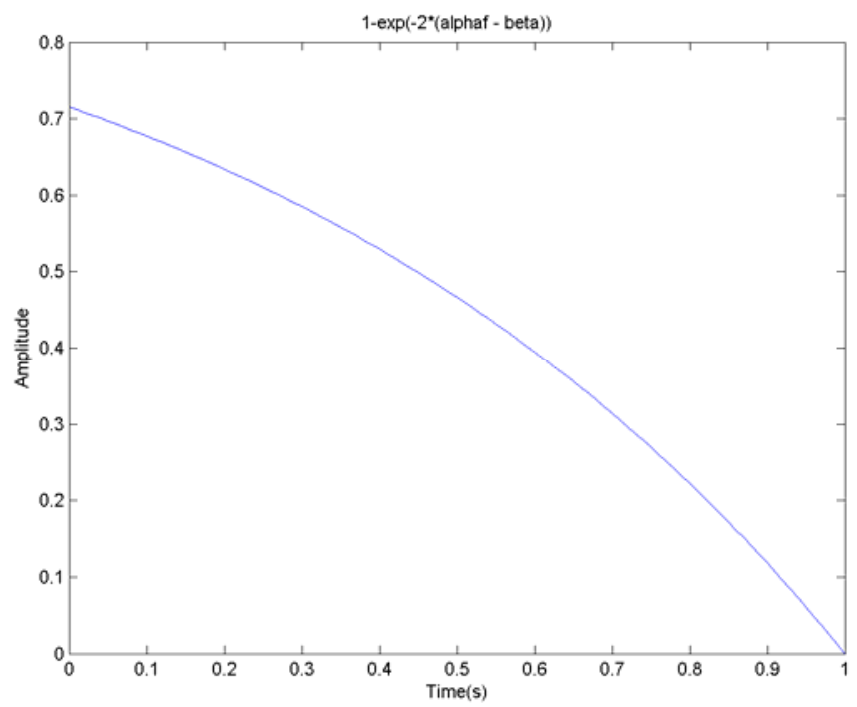

Figure 11. Numerical Scaling Factor for Autocorrelation Function based on a One Second Time History 
As the final time gets larger, the resulting function shown in Figure 11 rapidly approaches 1.0 for most lags in the correlation function. Figure 12 shows the function for a 10 second time history and Figure 13 for a 100 second data record. Hence for short time records the damping will be significantly affected by each correlation processing step that is used. For longer time histories, the numerical damping effects are limited to the last few lags of the resulting correlation function. However, these limited longer time history effects apparently build up over time to reach some steady-state value of numerical damping. It is very interesting that the modal frequency is largely unaffected by this numerical error.

\section{Recommended Follow-on Work}

The direct use of the suggested convergence metric for recursive correlation functions to extract modal damping is not a promising activity based on this work. Although, a more in-depth study of the damping modification build-up as a function of recursion step and the subsequent information contained in the phase of the analytic function are promising avenues to increase understanding. However, the ability clean up randomly excited data and extract well-behaved frequency estimates is an advantage to be pursued. A suggested next step would be to verify the frequency stability based on a larger set of increasingly realistic data characteristics as was mentioned above. If successful modal frequency extraction is seen, then these estimates could be used to drive a supplemental extraction process for damping. Such supplemental processes might be based on wavelet functions (see references 22 and 23 ) as a first recommended trial study.

\section{CONCLUSION}

The process of identifying modal parameters during launch is a difficult and challenging process. In this work, the launch phase of flight is assumed to be processed with small moving windows that are intended to keep the variations in modal parameters to a minimum such that stationary assumptions can hold. A more specific assumption of this work is that acceleration data in each window are processed into cross- and autocorrelation functions. It has been found that this process is hampered by "beating" or "blooms" in the correlation functions. These unwanted features limit the amount of data that can be used in the subsequent time domain algorithm processing. In typical ground based applications, these effects are rarely seen as frequency-domain averaging using long stationary time histories is highly effective at eliminating the issue.

An alternative approach has been reported that may be applicable in launch processing is the use of multiple iterations of correlation processing using the same base data. This mitigation had been hampered until recently by the lack of a convergence metric to allow the process to terminate. The recent development of a convergence metric has allowed this recursive correlation process to be subjected to further scrutiny. The convergence metric utilized a Hilbert transform of the resulting correlation function from each recursive step to generate an analytic representation of the data. The envelope of each correlation function can thus be extracted from the analytic function. The natural $\log$ of this envelope is then used to set the data into a format where a linear slope can be estimated. A significant finding is that for a limited class of data studied to-date (single dominant mode) is that these slope parameter estimates converge. Previously, this convergence seemed to produce an estimate of the proper damping for long enough time histories with a deterministic bias affecting shorter time histories. 


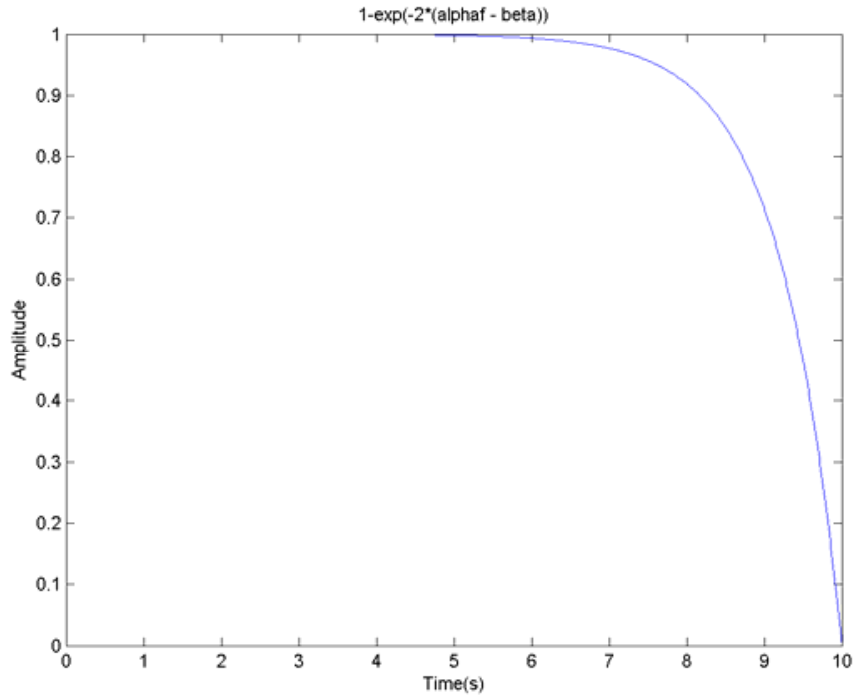

Figure 12. Numerical Scaling Factor for Autocorrelation Function based on a Ten Second Time History

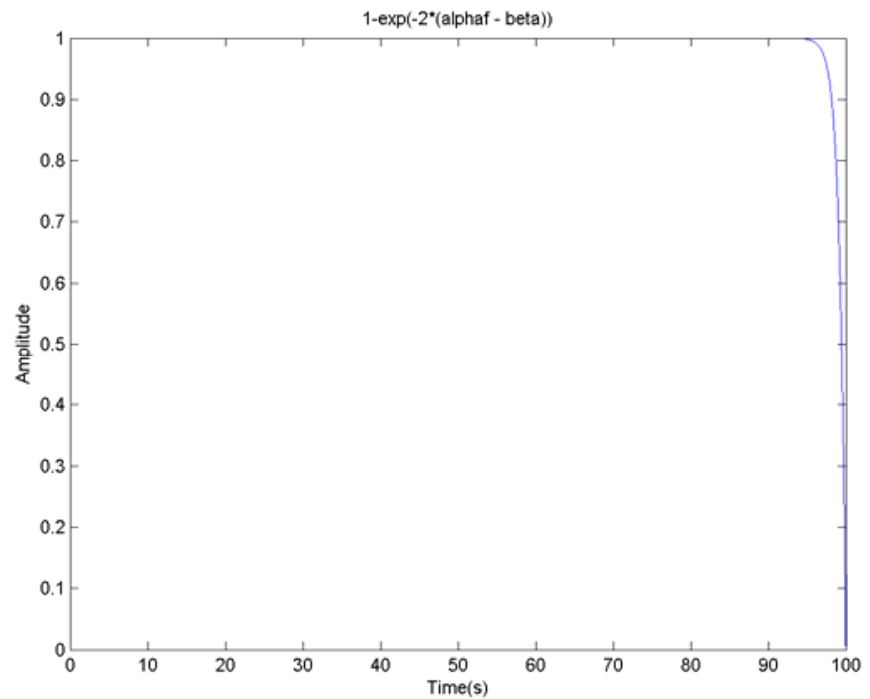

Figure 13. Numerical Scaling Factor for Autocorrelation Function based on a One Hundred Second Time History 
Work reported herein has shown that the proper modal damping is replaced with a fairly consistent value of numerical damping by repeated correlations. However, the modal frequency is left intact in this process. Subsequent mathematical studies have suggested that there is an expected numerical modification of the modal damping when short time history correlation processing is used. Furthermore this study has suggested that longer time histories will also be subjected to numerical damping modification via repeated correlation processing based on the same limitations of the correlation functions. There are some fruitful follow-on studies that can be envisioned to further understand the numerical effects on damping such as a mathematical assessment of multiple correlations and the information available in the phase of the analytical function.

The modal frequency estimation process becomes a much more refined activity and this advantage needs to be studied and pursued. If this holds after subsequent studies with more realistic data sets, then a supplemental process to extract damping can be pursued with refined estimates of frequency available. Wavelet technology has been suggested as one potential basis for such a supplemental process.

\section{APPENDIX A: THEORETICAL BASIS FOR AN OMA PROCESS, NEXT}

A critical step in the development of NExT was to find a function that could be measured from operational data, but possessed a clear relationship with and a dependence on the modal parameters of the structure. For NExT, the function selected was crosscorrelation functions between responses without a measurement of the input force. This section outlines the development of the relationship between the cross-correlation function and modal parameters. The full details of this development can be found in multiple references. ${ }^{1,40,41}$

The derivation begins by assuming the standard matrix equations of motion:

$$
[\mathrm{M}]\{\ddot{x}(\mathrm{t})\}+[C]\{\dot{x}(\mathrm{t})\}+[\mathrm{K}]\{\mathrm{x}(\mathrm{t})\}=\{f(t)\}
$$

where

$[\mathrm{M}]$ is the mass matrix;

$[\mathrm{C}]$ is the damping matrix;

$[\mathrm{K}]$ is the stiffness matrix;

$\{\mathrm{f}\}$ is a vector of random forcing functions;

$\{\mathrm{x}\}$ is the vector of random displacements; and $\mathrm{t}$ is time.

Equation (1) can be expressed in modal coordinates using the standard modal transformation and diagonalized matrices (assuming proportional damping). A solution to the resulting scalar modal equations can be performed via the convolution or a Duhamel integral and assuming a general forcing function $\{f\}$ with zero initial conditions. ${ }^{42}$ The solution can be converted back into physical coordinates and specialized for a single input force and a single output using appropriate mode shape matrix entries. The following equation results: 


$$
\mathrm{x}_{\mathrm{ik}}(\mathrm{t})=\sum_{\mathrm{r}=1}^{\mathrm{n}} \psi_{\mathrm{ir}} \psi_{\mathrm{kr}} \cdot \int_{-\infty}^{\mathrm{t}} \mathrm{f}_{\mathrm{k}}(\tau) \mathrm{g}^{\mathrm{r}}(\mathrm{t}-\tau) \mathrm{d} \tau
$$

where

$$
g r(t)=0, \text { for } t<0 ; g r(t)=\frac{1}{m^{r} \omega_{d}^{r}} \exp \left(-\zeta r \omega_{n}^{r} t\right) \sin \left(\omega{ }_{d}^{r} t\right), \text { for } t \geq 0
$$

$\omega_{\mathrm{d}}^{\mathrm{r}}=\omega_{\mathrm{n}}^{\mathrm{r}}\left(1-\zeta^{2}\right)^{1 / 2}$ is the damped modal frequency;

$\omega_{\mathrm{n}}^{\mathrm{r}}$ is the $r^{\text {th }}$ modal frequency;

$\zeta^{\mathrm{r}}$ is the $r^{\text {th }}$ modal damping ratio;

$\mathrm{m}^{\mathrm{r}}$ is the $r^{\text {th }}$ modal mass;

$\mathrm{n}$ is the number of modes;

$\psi_{\text {ir }}$ is the $i^{\text {th }}$ component of mode shape $\mathrm{r}$; and

$\mathrm{t}$ is the time.

The next step of the theoretical development is to form the cross-correlation function of two responses $\left(\mathrm{x}_{\mathrm{ik}}\right.$ and $\left.\mathrm{x}_{\mathrm{jk}}\right)$ due to a white-noise input at a particular input point $\mathrm{k}$. The cross-correlation function $\Re$ as the expected value of the product of two responses evaluated at a time separation of $\mathrm{T}$,

$$
\Re_{\mathrm{ijk}}(\mathrm{T})=\mathrm{E}\left[\mathrm{x}_{\mathrm{ik}}(\mathrm{t}+\mathrm{T}) \mathrm{x}_{\mathrm{jk}}(\mathrm{t})\right]
$$

where $\mathrm{E}$ is the expectation operator. ${ }^{13,43}$

Substituting Equation (2) into (3) and recognizing that the force $f$ is the only random variable, then the expectation operator functions only on the forcing function. Using the definition of the autocorrelation function ${ }^{17}$, and assuming for simplicity that the forcing function is white noise (this is only approximately true), then the expectation operation collapses to a scalar times a Dirac delta function. The Dirac delta function collapses one of the Duhamel integrations embedded in the cross-correlation function. The resulting equation can be simplified via a change of variable of integration $(\lambda=t-\tau)$. Using the definition of $g$ from Equation (2) and the trigonometric identity for the sine of a sum results in all the terms involving $\mathrm{T}$ being separated from those involving $\lambda$. This separation allows terms that depend on $\mathrm{T}$ to be factored out of the remaining integral and out of one of the modal summations. This results in: 


$$
\mathfrak{R}_{i j k}(T)=\sum_{r=1}^{n}\left[A_{i j k}^{r} \exp \left(-\zeta^{\mathrm{r}} \omega_{\mathrm{n}}^{\mathrm{r}} T\right) \cos \left(\omega_{\mathrm{d}}^{\mathrm{r}} T\right)+B_{i j k}^{r} \exp \left(-\zeta^{\mathrm{r}} \omega_{\mathrm{n}}^{\mathrm{r}} T\right) \sin \left(\omega_{\mathrm{d}}^{\mathrm{r}} T\right)\right]
$$

where $\mathrm{A}_{\mathrm{ijk}}^{\mathrm{r}}$ and $\mathrm{B} \underset{\mathrm{ijk}}{\mathrm{r}}$ are independent of $\mathrm{T}$, are functions of only the modal parameters, contain completely the remaining modal summation, and are shown below.

$$
\left\{\begin{array}{c}
A_{\mathrm{ijk}}^{\mathrm{r}} \\
B_{\mathrm{ijk}}^{\mathrm{r}}
\end{array}\right\}=\sum_{\mathrm{s}=1}^{\mathrm{n}} \frac{\alpha_{\mathrm{k}} \psi_{\mathrm{ir}} \psi_{\mathrm{kr}} \psi_{\mathrm{js}} \psi_{\mathrm{ks}}}{\mathrm{m}^{\mathrm{r}} \omega_{\mathrm{d}}^{\mathrm{r}} \mathrm{m}^{\mathrm{s}} \omega_{\mathrm{d}}^{\mathrm{s}}} \cdot \int_{0}^{\infty} \exp \left(-\zeta^{\mathrm{r}} \omega_{\mathrm{n}}^{\mathrm{r}}-\zeta^{\mathrm{s}} \omega_{\mathrm{n}}^{\mathrm{s}}\right) \lambda \cdot \sin \left(\omega_{\mathrm{d}}^{\mathrm{s}} \lambda\right)\left\{\begin{array}{c}
\sin \left(\omega_{\mathrm{d}}^{\mathrm{r}} \lambda\right) \\
\cos \left(\omega_{\mathrm{d}}^{\mathrm{r}} \lambda\right)
\end{array}\right\} \mathrm{d} \lambda
$$

Equation (4) is now the key result of this derivation because many time-domain modal analysis algorithms utilized impulse response functions as the input data for estimating the modal parameters. We see that Equation (4) shows that the cross-correlation function has the same characteristics as the impulse response function, a sum of decaying sinusoids with the same damping and frequency as the impulse response function. Thus, cross-correlation functions can be used in place of impulse response functions in these time-domain modal parameter estimation algorithms. Consequently, Equation (4) provides us with the desired function that can be measured from operational data and used to extract the modal parameters. The reader can refer to the provided references for more details of the intermediate steps in this derivation. ${ }^{1,14,15}$ These references also provide verification of this derivation, using the maximum of an autocorrelation function and a cross-correlation identity.

\section{REFERENCES}

${ }^{1}$ G. James, T. Carne, and J. Lauffer, "The Natural Excitation Technique (NExT) for Modal Parameter Extraction from Operating Structures”. SEM International Journal of Analytical and Experimental Modal Analysis, Vol. 10, No. 4. 1995.

${ }^{2}$ H. Vold and T. Rocklin, “The Numerical Implementation of a Multi Input Modal Estimation Algorithm for Mini-Computers", Proceedings of IMAC I, Orlando, FL, 1982.

${ }^{3}$ J.-N. Juang and R. Pappa, “An Eigensystem Realization Algorithm for Modal Parameter Identification and Model Reduction”, AIAA Journal of Guidance, Control and Dynamics, Vol. 8, N. 4, 1985, p. 620-627.

${ }^{4}$ R. Brinker and P. Kirkegaard, Editorial in the special issue on operational modal analysis, Mechanical Systems and Signal Processing, Vol. 24, No. 5, 2010, pp. 1209-1212.

${ }^{5}$ L. Hermans and H. Van der Auweraer, "On the Use of Auto and Cross-Correlation Functions to Extract Modal Parameters from OutputOnly Data”, Proceedings of Structural Dynamics: Recent Advances, Southhampton, U.K., July 14-17, 1997.

${ }^{6}$ T. Cao, D. Zimmerman, and G. James, "Identification of Ritz Vectors from Ambient Test Data”, Proceedings of IMAC XVI, Santa Barbara, CA, February 2-5, 1998. 
${ }^{7}$ L. Zhang, R. Brinker, and P. Andersen, "Modal Indicators for Operational Modal Identification”, Proceedings of IMAC XIX. Kissimmee, FL, 2001.

${ }^{8}$ P. Mohanty and D. Rixen, "Modifying the ERA Identification for Operational Modal Analysis in the Presence of Harmonic Perturbations", Proceedings of the $16^{\text {th }}$ ASCE Engineering Mechanics Conference, Seattle, July 16-18, 2003.

${ }^{9}$ J. Calcedo, S. Dyke, and E. Johnson, "Natural Excitation Technique and Eigensystem Realization Algorithm for Phase I of the IASCASCE Benchmark Problem: Simulated Data", Journal of Engineering Mechanics, Vol. 130, No. 1, 2004.

${ }^{10}$ D-Y Chiang and C-S Lin, "Identification of Modal Parameters from Nonstationary Ambient Vibration Data Using Correlation Technique”, Proceedings of IMAC XXX. Jacksonville, FL, Jan. 30 - Feb. 2, 2012.

${ }^{11}$ T. Carne and G. James, "The Inception of OMA in the Development of Modal Testing Technology for Wind Turbines", Mechanical Systems and Signal Processing, Vol. 24, No. 5, 2010, pp. 1213-1226.

${ }^{12}$ J. Brownjohn and P. Carden, "Reliability of Frequency and Damping Estimates from Free Vibration Response", Proceedings of the $2^{\text {nd }}$ International Operational Modal Analysis Conference, Copenhagen, Denmark, 2007, pp. 23-30.

${ }^{13}$ L. Zhang, T. Wang T, and Y. Tamura, “A Frequency-Spatial Domain Decomposition (FSDD) Method for Operational Modal Analysis”, Mechanical Systems and Signal Processing, Vol. 24, No. 5, 2010, pp. 1227-1239.

${ }^{14}$ F. Magalhăes, A. Cunha, E. Caetano, and R. Brinker, "Damping Estimation Using Free Decays and Ambient Vibration Tests", Mechanical Systems and Signal Processing, Vol. 24, No. 5, 2010, pp. 1274-1290.

${ }^{15}$ R. Campbell and J. Vandiver, "The Estimation of Natural Frequencies and Damping Ratios of Offshore Structures”, Proceedings of the $12^{\text {th }}$ Annual Offshore Technology Conference, Houston, TX, 1980.

${ }^{16}$ M. Briggs, "Multichannel maximum entropy method of spectral analysis applied to offshore structures", WHOI-81-69, Woods Hole Oceanographic Institute, 1981.

${ }^{17}$ S. Ibrahim, "Random Decrement Technique for Modal Identification of Structures", Journal of Spacecraft and Rockets, Vol. 14, N. 4, 1977, pp. 183-198.

${ }^{18}$ J. Asmussen, S. Ibrahim, and R. Brinker, "Random Decrement: Identification of Structures Subjected to Ambient Excitation", Proceedings of IMAC XVI, Santa Barbara, CA, Feb. 2-5, 1998.

${ }^{19}$ A. Agneni, L.B. Crema, and G. Coppotelli, "Output-only Analysis of Structures with Closely Spaced Poles", Mechanical Systems and Signal Processing, Vol. 24, No. 5, 2010, pp. 1241-1249.

${ }^{20}$ I. Bjerg, S. Hansen, R. Brinker, and M.L. Aenlle, "Load Estimation by Frequency Domain Decomposition", Proceedings of the $2^{\text {nd }}$ International Operational Modal Analysis Conference, Copenhagen, Denmark, 2007, pp 669-676.

${ }^{21}$ G. James, T. Carne, and B. Wilson, "Reconstruction of the Space Shuttle Roll-out Forcing Function", Proceedings of the IMAC XXV, Orlando, FL, Feb. 19-23, 2007,

${ }^{22}$ L. Freudinger, R. Lind, and M. Brenner, “Correlation Filtering of Modal Dynamics using the Laplace Wavelet”, NASA CR-97-207066. 1997.

${ }^{23}$ R. Lind, M. Brenner, and S. Haley “Estimation of Modal Parameters Using a Wavelet Approach”, NASA TM-97-206300, 1997.

${ }^{24}$ K. Gröchenig, Foundations of Time-Frequency Analysis, Birkhäuser, 2001, p. 386. 
${ }^{25}$ N.E. Huang, et al., "The empirical mode decomposition and the Hilbert spectrum for non-linear and non-stationary time series analysis", Proceedings of the Royal Society of London, Vol. 454, 1998, pp. 903-993.

${ }^{26}$ J. Chen and Y.L. Xu, "Identification of modal damping ratios of structures with closely spaced modal frequencies", Structural Engineering and Mechanics, Vol. 14, No. 4, 2002, pp. 417-434.

${ }^{27}$ Y.L. Xu, S.W. Chen, R C. Zhang, "Modal Identification of Di Wang Building under Typhoon York using the Hilbert-Huang Transform Method", The Structural Design of Tall and Special Buildings, Vol. 12, No. 1, 2003, pp. 21-47.

${ }^{28}$ G. James, T. Carne, and E. Marek, "In-situ Modal Analysis of STARS Missile Flight Data and Comparison to Pre-Flight Predictions from Test-Reconciled Models", Proceedings of the 15th IES Aerospace Testing Seminar, Manhattan Beach, CA, October 11-13, 1994.

${ }^{29}$ G. James, "Modal Parameter Estimation from Space Shuttle Flight Data”, Proceedings of IMAC XXI, Kissimmee, FL, Feb. 3-6, 2003.

${ }^{30}$ G. James, et. al., "Operational Analysis in the Launch Environment”, Proceedings of IMAC XXX, Jacksonville, FL, Jan. 30-Feb. 2, 2012.

${ }^{31}$ H. Kim, D. Van Horn, and H. Doiron, "Free-decay Time-Domain Modal Identification for Large Space Structures", Journal of Guidance, Control, and Dynamics, Vol. 17, No. 3, 1994, pp. 513-519.

${ }^{32}$ V. Le Gallo, M. Goursat, and L. Gonidou, “Damping Characterization and Flight Identification”, Proceedings of IMAC XXV, Orlando, FL, 2007.

${ }^{33}$ M. Goursat, M. Döhler, L. Mevel, and P. Andersen, "Crystal Clear SSI for Operational Modal Analysis of Aerospace Vehicles”, Proceedings of IMAC XXVIII, Jacksonville, FL, 2010.

${ }^{34}$ S. Fransen, D. Rixen, T. Henricksen, and M. Bonnet M, "On the Operational Modal Analysis of Solid Rocket Motors", Proceedings of IMAC XXVIII, Jacksonville, FL, 2010.

${ }^{35}$ T. Bartkowicz and G. James, “Ares 1-X In-Flight Modal Identification”, Proceedings of $52^{\text {nd }}$ AIAA/ASME/AHS/ASC SDM Conference, Boulder, CO, April 4-8. 2011.

${ }^{36}$ G. James, K. Chhipwadia K, and D. Zimmerman, “Application of Autonomous Modal Identification to Traditional and Ambient Data Sets. Proceedings of IMAC XVII, 1999.

${ }^{37}$ R. Pappa, G. James, and D. Zimmerman, “Application of Autonomous Modal Identification of the Space Shuttle Tail Rudder”, AIAA Journal of Spacecraft and Rockets, Vol. 35, No. 2, 1999, pp. 163-169.

${ }^{38}$ G. James, Development of Operational Modal Analysis Techniques for Launch Data, AAS-12-627, American Astronautical Society, 2012.

${ }^{39}$ G. James, T. Cao, V. Fogt, R.L. Wilson, and T. Bartkowitz, "Extraction of Modal Parameters from Spacecraft Flight Data”, Proceedings of IMAC XXIX, Jacksonville, FL, 2011

${ }^{40}$ G.H. James, T.G. Carne, J.P. Lauffer, "The Natural Excitation Technique (NExT) for Modal Parameter Extraction From Operating Wind Turbines", SAND92-1666, Sandia National Laboratories, Albuquerque, NM, USA,1993.

${ }^{41}$ G.H. James, T.G. Carne, J.P. Lauffer, A.R. Nord, "Modal Testing Using Natural Excitation", Proceedings of IMAC X, San Diego, California, Feb. 3-7, 1992.

42 S.H. Crandall, Random Vibration, John Wiley \& Sons, Inc., New York, USA, 1958.

43 A. Papoulis, Probability, Random Variables, and Stochastic Processes, McGraw-Hill, New York, NY, 1965. 